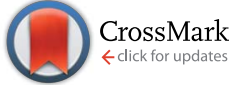

Cite this: RSC Adv., 2017, 7, 12179

Received 19th December 2016 Accepted 5th February 2017

DOI: 10.1039/c6ra28384e

rsc.li/rsc-advances

\section{Influence of interface interaction on the moiré superstructures of graphene on transition-metal substrates}

\author{
Lei Gao, Yanmin Liu, Ruoyu Shi, Tianbao Ma, ${ }^{*}$ Yuanzhong Hu and Jianbin Luo
}

The formation of moiré superstructures between graphene and its underlying substrate has attracted significant attention because it significantly influences the morphology and properties of graphene. Through the density functional theory (DFT) calculations conducted on graphene/Re(0001) and graphene/Ir(111) moiré superstructures, we found that in contrast to the strain-driven moiré superstructure of graphene on weak van der Waals interacting $\operatorname{Ir}(111)$ substrate, the interfacial interaction dominates in the moiré superstructure of graphene on the covalently interacting $\operatorname{Re}(0001)$ substrate. A large strain is exerted on graphene to facilitate the interfacial interaction between graphene and $\operatorname{Re}(0001)$ substrate, which markedly reduces the interfacial interaction energy and stabilizes the graphene/Re(0001) moiré superstructure. The strong covalent interaction between graphene and $\operatorname{Re}(0001)$ substrate is closely related to the hybridization between $C 2 p_{z}$ orbital and $\operatorname{Re} 5 d_{z^{2}}$ orbital, which is absent in the weak van der Waals interacting graphene/Ir(111) moiré superstructure.

\section{Introduction}

Graphene grown on transition-metal substrates forms various moiré superstructures due to the lattice mismatch between graphene and the substrates. Moiré superstructures are important for the occurrence of novel electronic, mechanical, and catalytic properties in graphene; thus, their growth, structure, and stability have been extensively studied..$^{1-6}$ To date, the formation of moiré superstructures of graphene epitaxially grown on different transition-metal substrates, such as $\operatorname{Ir}^{7,8}$ $\mathrm{Pt},{ }^{9,10} \mathrm{Au},{ }^{11} \mathrm{Ru},{ }^{12-16}$ and $\mathrm{Re},{ }^{17,18}$ showing different periodicities and orientations, has been reported and usually more than one configurations have been reported for a certain substrate. Moiré superstructures of graphene on $\operatorname{Ir}(111)$ with different orientations have been systematically studied by experimental observations and theoretical calculations to show that the weak van der Waals interactions between graphene and the $\operatorname{Ir}(111)$ substrate lead to the coexistence of multi-oriented moiré superstructures. ${ }^{7}$ The appearance of a particular periodicity of moiré superstructure in the multi-domain epitaxial graphene on $\mathrm{Pt}(111)$ substrate is found to be driven by the minimization of the absolute value of the strain between graphene and the substrate lattice. ${ }^{19}$ In contrast, the moire superstructure shows perfect alignment between graphene and the $\mathrm{Ru}(0001)$ substrate. ${ }^{\mathbf{1 2 - 1 6}}$ The strong interaction between $\mathrm{C}$ atoms and $\mathrm{Ru}$ atoms results in the stable configuration of a $3 \mathrm{~nm}$ moiré superstructure, which maximizes the interfacial $\mathrm{C}-\mathrm{Ru}$ bonding

State Key Laboratory of Tribology, Tsinghua University, Beijing 100084, China. E-mail: mtb@mail.tsinghua.edu.cn and interfacial adhesion, whereas minimizes the lattice mismatch. ${ }^{15}$ Recently, the atomic structure of graphene on $\operatorname{Re}(0001)$ substrate has also been experimentally studied, showing two stable moiré superstructures of $(8 \times 8)$ graphene unit cells over $(7 \times 7) \operatorname{Re}(0001)$ unit cells $(8 \times 7$ model $)$ and $(10$ $\times 10)$ graphene unit cells over $(9 \times 9) \operatorname{Re}(0001)$ unit cells $(10 \times 9$ model). ${ }^{17,18}$ To elucidate the driving force for the various moiré superstructures, the interplay or competition between the strain energy and interfacial interaction should be studied in depth.

In this study, density functional theory (DFT) calculations were carried out to investigate the two experimentally reported stacking of graphene/Re(0001) moiré superstructures to reveal the effects of interfacial interaction on the structure and energy stability of graphene on the $\operatorname{Re}(0001)$ substrate. On comparing with the graphene/Ir(111) moiré superstructure, we found that the intensive covalent interaction between graphene and the $\operatorname{Re}(0001)$ substrate can impose a much larger strain on graphene to facilitate the interaction between graphene and the $\operatorname{Re}(0001)$ substrate. The strong covalent interfacial interaction is closely related to the hybridization between the $\mathrm{C} 2 \mathrm{p}_{z}$ orbital and $\operatorname{Re} 5 \mathrm{~d}_{z^{2}}$ orbital in the flat region.

\section{Calculation methods}

The DFT calculations were implemented using the Vienna ab initio simulation Package (VASP). ${ }^{20}$ The projector-augmentedwave (PAW) method was utilized to model the core electrons. A non-local optB86b-vdW exchange-correlation functional ${ }^{21,22}$ was used due to its ability to approximately describe the dispersion interaction (van der Waals forces) and it has been 
demonstrated to be currently the most accurate functional among the vdW functionals. ${ }^{23}$ The plane wave basis kinetic energy cut-off was set at $400 \mathrm{eV}$.

All the graphene/Re(0001) (both the $8 \times 7$ supercell and the $10 \times 9$ supercell) and graphene/Ir(111) supercells contain three layers of substrate lattices and a graphene layer with a vacuum slab of more than $21 \AA$. Previous DFT calculations indicated that three layers of substrate lattices are adequate to describe the moiré superstructures of graphene on transition-metal substrates, ${ }^{9,15,16}$ providing computational efficiency, whereas slightly sacrificing accuracy while describing the electronic properties as compared to the calculations for more layers of substrate lattices. ${ }^{\mathbf{2 4 2 5}}$ Graphene and first substrate layer were allowed to relax until the forces on all the relaxed atoms were less than $0.02 \mathrm{eV}^{-1}$. All the calculations were carried out using relaxed Re lattice constants ( $a=2.767 \AA$ and $c=4.466 \AA)$, Ir lattice constant $(a=3.865 \AA)$, and graphene lattice constant $(a=$ $2.464 \AA)$. To accommodate the monolayer graphene and $\operatorname{Re}(0001)$ substrate in one commensurate calculation supercell, compulsive compressive $(1.75 \%)$ and tensile $(1.06 \%)$ strains were applied on graphene in the $8 \times 7$ model and in the $10 \times 9$ model, respectively. Due to the large sizes of the considered calculation supercells, the Brillouin zone was sampled with a single $k$-point at $\Gamma$ during the geometrical relaxation; then, the density of states was calculated with a dense $6 \times 6 \times 1$ $k$-sampling to describe a more accurate electronic structure.

\section{Results and discussion}

Fig. 1 shows the atomic stacking of graphene and the top layer of the $\operatorname{Re}(0001)$ substrate in the $8 \times 7$ and $10 \times 9$ models after relaxation. The color on the $\mathrm{C}$ atoms reflects the morphology corrugation of graphene. In Fig. 1, the formation of moire superstructure in both the $8 \times 7$ and $10 \times 9$ models can be clearly observed. The periodicities of the moiré superstructures in the $8 \times 7$ model and in the $10 \times 9$ model were $1.94 \mathrm{~nm}$ and $2.49 \mathrm{~nm}$, respectively. Moreover, the vertical humps were formed in the moire superstructures and were surrounded by the flat regions, where the $\mathrm{C}$ atoms and Re atoms couple with the head to head stacking. Comparing the morphologies of graphene in the $8 \times 7$ model and in the $10 \times 9$ model, it can be observed that the corrugation of the hump in the $8 \times 7$ model is larger than that in the $10 \times 9$ model, whereas the proportion of the flat region in the $8 \times 7$ model is obviously smaller than that in the $10 \times 9$ model. The calculated morphologies of the two moire superstructures are consistent with the previous experimental observations. ${ }^{17,18}$ To investigate the stability of the two moire superstructures, the interaction energy between graphene and $\operatorname{Re}(0001)$ substrate and the strain energy in graphene were analyzed.

As an indicator to reflect the interaction strength between graphene and the $\mathrm{Re}(0001)$ substrate, the distances between $\mathrm{C}$ atoms in graphene and the $\operatorname{Re}(0001)$ substrate were calculated in the $8 \times 7$ model and in the $10 \times 9$ model and are shown in Fig. 2a. In the $10 \times 9$ model, nearly half of the $\mathrm{C}$ atoms in graphene have a smaller distance to the $\operatorname{Re}(0001)$ substrate, ranging from 2.2 to $2.3 \AA$; however, there is a lack of a certain preferential distance between graphene and the $\operatorname{Re}(0001)$ substrate in the $8 \times 7$ model. The interaction energies between graphene and the $\operatorname{Re}(0001)$ substrate in the two models were calculated using eqn (1) and indicated that the interaction energy in the $10 \times 9$ model is indeed lower than that in the $8 \times$ 7 model, as shown in Fig. 2 b.

$$
E_{\text {interaction }}=\left(E_{\mathrm{Gr} / \mathrm{Re}}-E_{\mathrm{Re}}-E_{\mathrm{Gr}}\right) / S
$$

where $E_{\mathrm{Gr} / \mathrm{Re}}, E_{\mathrm{Re}}$, and $E_{\mathrm{Gr}}$ are the energy of the graphene/ $\operatorname{Re}(0001)$ moiré superstructure, and configuration energies of the separated $\operatorname{Re}(0001)$ substrate and graphene with the same configurations as that of their structures in graphene/Re(0001) moiré superstructure, respectively. $S$ represents the area of the interface between graphene and the $\operatorname{Re}(0001)$ substrate.

The charge-transfer distributions between graphene and the $\operatorname{Re}(0001)$ substrate could help to reveal the causes of the stronger interfacial interaction intensity in the $10 \times 9$ model. Fig. $2 c$ and d show the charge-transfer distributions in the (001) planes of the $8 \times 7$ and $10 \times 9$ models, and the charge-transfer intensities at the flat region in the two models confirm that their interactions are in the range of covalent interactions. Comparing Fig. 1, Fig. 2c and d, we found more intensive charge-transfer sites in the $10 \times 9$ model than those in the $8 \times 7$ model, which is due to the larger proportion of the flat region where the $\mathrm{C}$ atoms and $\mathrm{Re}$ atoms couple in a head-to-head stacking fashion in the $10 \times 9$ model.

Besides the interfacial interaction energy, as suggested by the observation of giant vertical humps in the graphene/

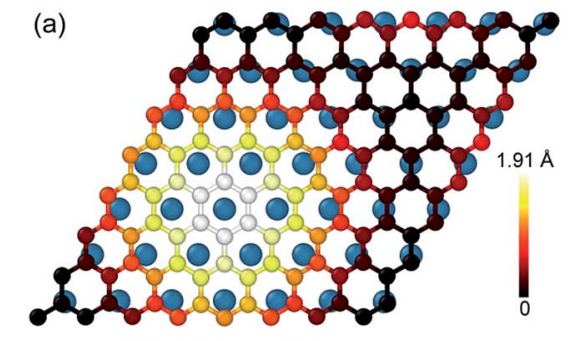

$\mathrm{Gr} / \mathrm{Re}(0001) 8 \times 7$

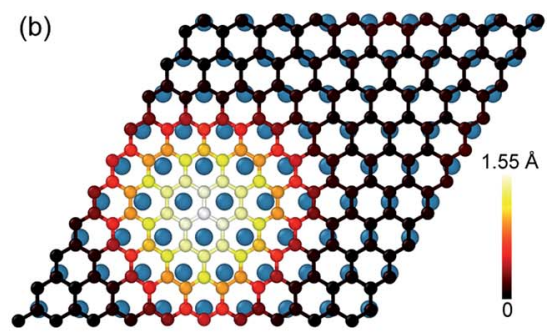

$\mathrm{Gr} / \operatorname{Re}(0001) 10 \times 9$

Fig. 1 Comparison of the morphologies of graphene on the Re(0001) substrate in the $8 \times 7$ model and the $10 \times 9$ model. (a) and (b) Illustrate the geometrical atomic stacking between graphene and the top layer of the Re(0001) substrate in the $8 \times 7$ model and the $10 \times 9$ model. The color on the $\mathrm{C}$ atoms reflects the corrugation of graphene. 


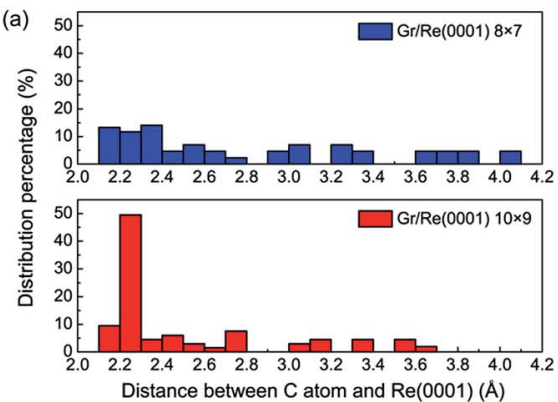

(c) $\operatorname{Gr} / \operatorname{Re}(0001) 8 \times 7$

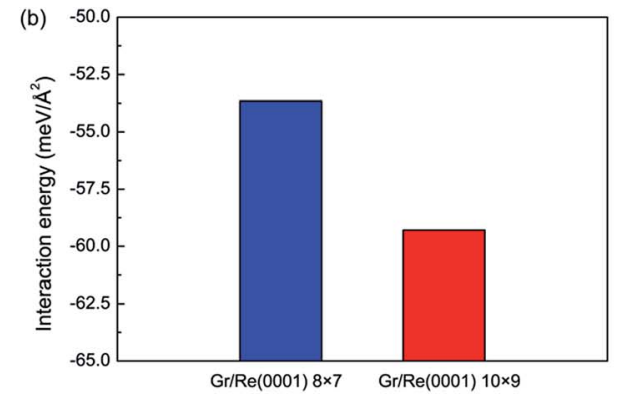

(d) $\operatorname{Gr} / \operatorname{Re}(0001) 10 \times 9 \% \circ \circ \% \ldots$
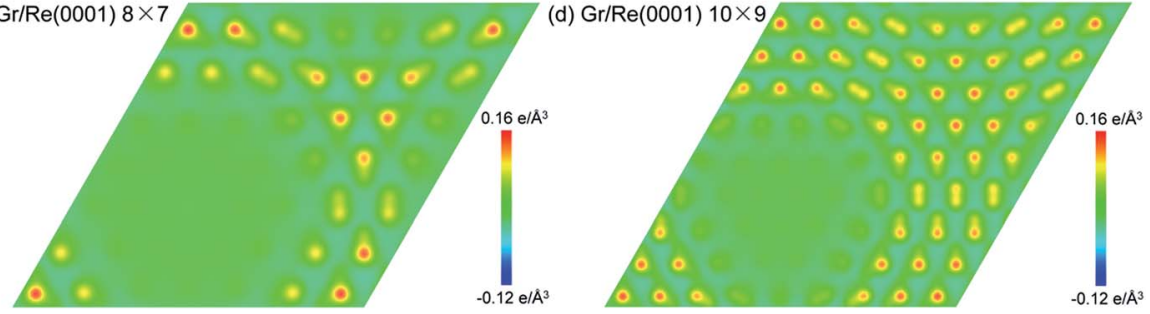

Fig. 2 Comparison of the interfacial interaction intensities between graphene and the Re(0001) substrate in the $8 \times 7$ model and $10 \times 9$ model. (a) The distribution of distances between $C$ atoms in graphene and Re(0001) substrate in the $8 \times 7$ model and $10 \times 9$ model. (b) The interaction energy between graphene and the $\operatorname{Re}(0001)$ substrate in the two models. (c) and (d) The charge transfer distributions in the (001) planes of the two models.

$\operatorname{Re}(0001)$ moiré superstructures, the strain energy in graphene should also significantly influence the moire superstructure stability. Although the hump height of graphene in the $8 \times 7$ model $(1.91 \AA)$ is larger than that in the $10 \times 9 \operatorname{model}(1.55 \AA)$, as shown in Fig. 1, the graphene strain energy in the $8 \times 7$ model is a bit smaller, as shown in Fig. 3a; herein, the graphene strain energy was obtained from the following equation:

$$
E_{\text {strain }}=\left(E_{\mathrm{Gr}}-E_{\mathrm{Gr} \text {-ideal }}\right) / S
$$

where $E_{\mathrm{Gr}}$ is the configuration energy of the separated graphene in the graphene/Re(0001) moire superstructure without relaxation, $E_{\text {Grideal }}$ is the energy of ideal graphene, and $S$ represents the area of the interface between graphene and the $\operatorname{Re}(0001)$ substrate.

From Fig. 3a, we found that the strain energies are quite large in the two models. The surprising larger graphene strain energy in the $10 \times 9$ model relative to that of the $8 \times 7$ model could be attributed to the relatively larger $\mathrm{C}-\mathrm{C}$ bond lengths, as
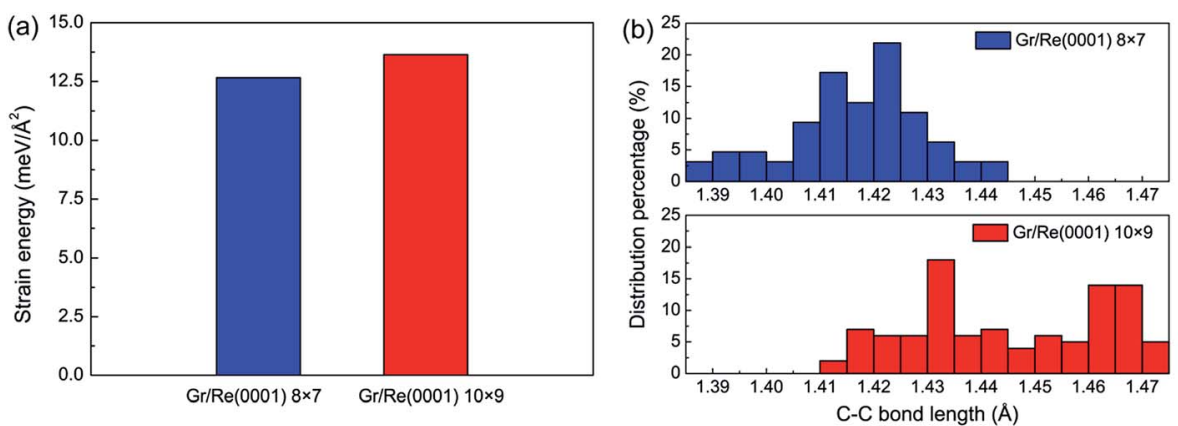

(c)

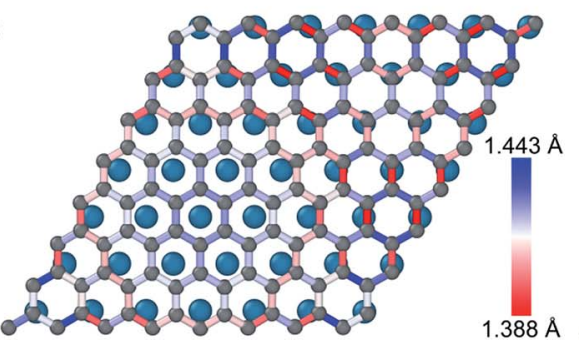

(d)

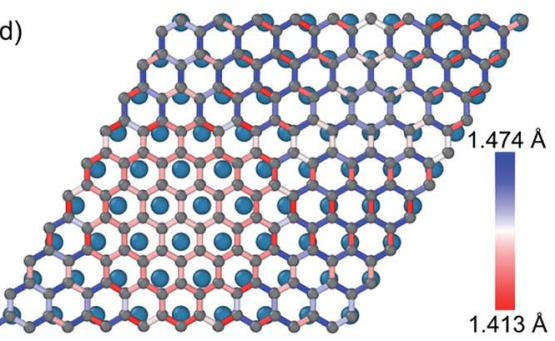

Fig. 3 (a) A comparison of the strain energies in graphene in the $8 \times 7$ model and $10 \times 9$ model. (b) The $C-C$ bond length distribution percentage in the two models. (c) and (d) are the detailed $\mathrm{C}-\mathrm{C}$ bond length distributions in the two models. 
illustrated in Fig. $3 \mathrm{~b}$. In the $8 \times 7$ model, the macro-strain in graphene prior to relaxation was $1.75 \%$ compressive. After relaxation, the hump formed and elongated the $\mathrm{C}-\mathrm{C}$ bond lengths in graphene to compensate the previous compressive strain; thus, most of the $\mathrm{C}-\mathrm{C}$ bond lengths resided around the $\mathrm{C}-\mathrm{C}$ bond length of graphene $(1.42 \AA)$. However, in the $10 \times 9$ model, the macro-strain in graphene prior to relaxation was $1.06 \%$ tensile. After relaxation, most of the $\mathrm{C}-\mathrm{C}$ bond lengths were further elongated, leading to the larger strain energy in graphene. The detailed $\mathrm{C}-\mathrm{C}$ bond length distributions are shown in Fig. $3 \mathrm{c}$ and $\mathrm{d}$.

Interestingly, we found that with the existence of large strain in the graphene, the $\mathrm{C}-\mathrm{C}$ bond length distributions spatially varied in the two models. At the flat region, the interaction and stacking between $\mathrm{C}$ atoms and Re atoms dominated the bond length distributions. The $\mathrm{C}-\mathrm{C}$ bonds tended to be stretched to facilitate the stacking of the $\mathrm{C}$ atoms and the Re atoms in headto-head fashion at the fcc and hep regions of graphene, as defined in previous reports, ${ }^{\mathbf{2 6}}$ to lower the interaction energy between graphene and the $\mathrm{Re}(0001)$ substrate, whereas the $\mathrm{C}-\mathrm{C}$ bond length evolutions at the hump of graphene in the two models were opposite. Since the interaction between graphene and the $\operatorname{Re}(0001)$ substrate was much weaker at the hump, the $\mathrm{C}-\mathrm{C}$ bonds tended to be close to that in free graphene. Due to the different compulsive strains imposed on the two models, the evolution of $\mathrm{C}-\mathrm{C}$ bond lengths will be opposite. The rest regions were the bridges between the hump and flat region and the bridges between the hcp and fcc regions, which were both compressed in the two models.

Previous experimental study has interpreted that the discrepancy in the $8 \times 7$ model and $10 \times 9$ model could originate from the different methods used to grow graphene in the two experiments. ${ }^{17}$ Our calculation results indicate that the interaction energy and strain energy in the two models change in the opposite tendency, which might explain the occurrence of both models in the experiments. The direct relationship between the growth conditions and atomic structure still requires further study.

Considering both the interaction energy between graphene and the $\operatorname{Re}(0001)$ substrate, and the strain energy in graphene, we found that they are deeply influenced by the interfacial interaction between graphene and the $\operatorname{Re}(0001)$ substrate in the two models. The deviation of $\mathrm{C}-\mathrm{C}$ bond lengths from ideal graphene will introduce a large strain in graphene; however, the strong covalent interaction can greatly reduce the interfacial interaction energy to compensate the increase in the strain energy in graphene. In our calculations, the difference in the interaction energy is more marked than that in the strain energy, and the overall energy of the $10 \times 9$ model is $4.66 \mathrm{meV}$ $\AA^{-2}$, which is more favored than that of the $8 \times 7$ model due to its superior geometrical stacking to enlarge the flat region.

With respect to the weak van der Waals interaction dominating in the graphene/Ir(111) moiré superstructure, the height of the hump in the graphene/Ir(111) moire superstructure (Fig. 4a) is much lower than that in the graphene/Re(0001) moiré superstructure, as shown in Fig. 1. Moreover, Fig. 4b shows that the interaction energy between graphene and the $\operatorname{Ir}(111)$ substrate is much higher than that in the graphene/ $\operatorname{Re}(0001)$ moiré superstructure (Fig. 2b) due to weak charge transfer between them, as shown in Fig. 4c. However, the strain energy in graphene (Fig. 4b) is extremely lower than that in the graphene/ $\operatorname{Re}(0001)$ moiré superstructure (Fig. 3a); moreover, with the lack of strong interaction from the substrate, most of the $\mathrm{C}-\mathrm{C}$ bonds lengths in the graphene/Ir(111) moiré superstructure are close to those in the ideal graphene, as shown in Fig. $4 \mathrm{~d}$.

Comparing the graphene/Re(0001) and graphene/Ir(111) moiré superstructures, it is the interfacial interaction (namely the charge transfer) that determines the morphology and stability of graphene on the $\operatorname{Re}(0001)$ substrate. The charge

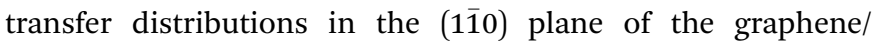
$\operatorname{Re}(0001)$ and graphene/Ir(111) moiré superstructures are shown in Fig. $5 \mathrm{a}$ and d, respectively. In Fig. 5a, it can be observed that the $\mathrm{C}$ atoms tended to interact with the Re atoms in the substrate; however, due to the atomic stacking difference in graphene, only the $\mathrm{C}$ atoms at the flat region could intensively interact with the $\operatorname{Re}(0001)$ substrate, whereas the region containing hexagonal holes of graphene over the Re atoms corrugates upwards to form the hump. In Fig. 5 d, it can be seen that the charge transfer intensities between $\mathrm{C}$ atoms and Ir atoms are quite weak at both the hump and the flat region, leading to the flattened morphology close to that of ideal graphene.

Previous studies illustrated that the strong interfacial interactions between graphene and transition metal substrates involve hybridization between $\mathrm{C} \pi$ orbitals and metal d orbitals, especially the hybridization between the $\mathrm{C} 2 \mathrm{p}_{z}$ orbital and the metal $\mathrm{d}_{z^{2}}$ orbital. ${ }^{15,16,24-28}$ The partial density of states (PDOS) of C atoms $\left(2 \mathrm{p}_{z}\right.$ orbital) and Re atoms $\left(5 \mathrm{~d}_{z^{2}}\right.$ orbital $)$ at the flat region and hump (labeled in Fig. 5a) helped to understand the charge transfer behavior between $\mathrm{C}$ atoms and $\mathrm{Re}$ atoms in the flat region. Fig. 5b confirms that the charge transfer behavior is due to the hybridization between the $\mathrm{C} 2 \mathrm{p}_{z}$ orbital and $\operatorname{Re} 5 \mathrm{~d}_{z^{2}}$ orbital, which is reflected in the correspondence of the PDOS curves (peak positions) between the two orbitals, as shown in Fig. $5 \mathrm{~b}$, indicating that the covalent interaction dominates at the flat region. However, at the hump, the PDOS curves of the C $2 \mathrm{p}_{z}$ orbital and $\operatorname{Re} 5 \mathrm{~d}_{z^{2}}$ orbital are independent, as shown in Fig. $5 \mathrm{c}$, which indicates hybridization did not occur. For the PDOS curves of the $\mathrm{C}$ atoms $\left(2 \mathrm{p}_{z}\right.$ orbital) and Ir atoms $\left(5 \mathrm{~d}_{z^{2}}\right.$ orbital) at both the flat region and the hump in the graphene/Ir(111) moiré superstructure, the hybridization is not observed, as shown in Fig. 5e and f. Thus, the hybridization between the $\mathrm{C} 2 \mathrm{p}_{z}$ orbital and the transition-metal $\mathrm{d}_{z^{2}}$ orbital corresponds to the strong covalent interaction between graphene and the substrate. This strong interfacial interaction further dominates the moire superstructure of graphene on the substrate. Even for the weak van der Waals interacting $\operatorname{Pt}(111)$ substrate, a recent study indicated that at the pinning-points, migration of the electronic charges from the $s$ towards the $d_{z^{2}}$ orbitals in the Pt atoms increases the orbital directionality, facilitating the hybridization with the $\mathrm{p}_{z}$ orbitals of the buckled graphene $\mathrm{C}$ atoms. ${ }^{29}$ By the proper selection and control of the interfacial interaction between graphene and the transition-metal substrate, a variety of moiré superstructures could be synthesized to meet the requirements of the practical applications. 

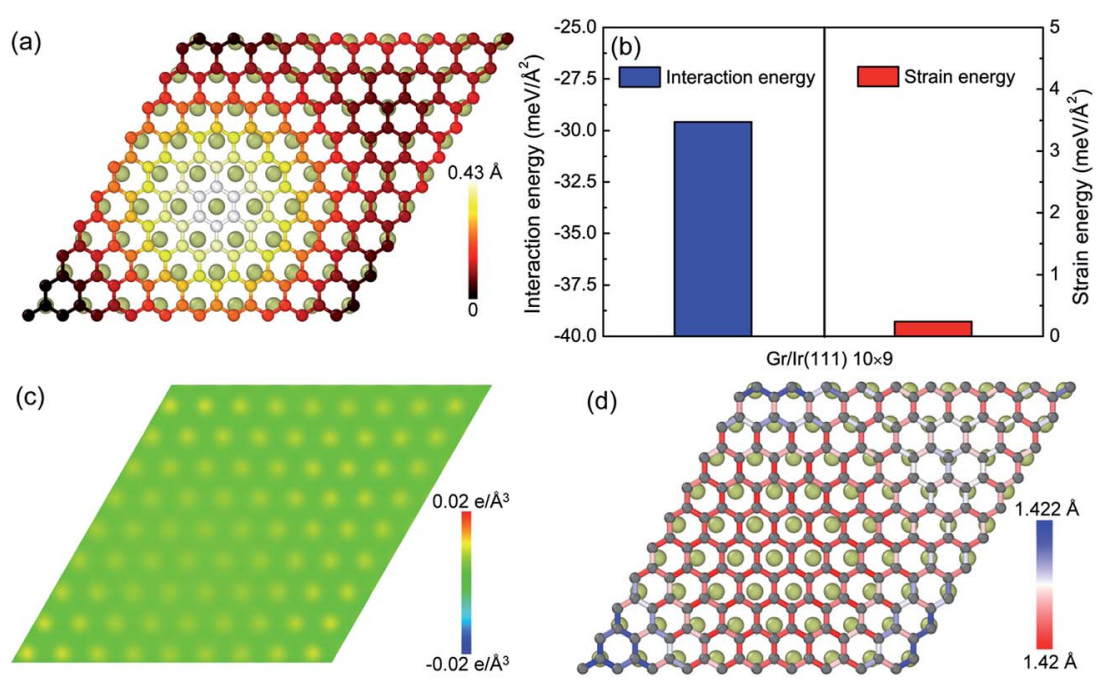

Fig. 4 (a) The geometrical atomic stacking between graphene and the top layer of the $\operatorname{Ir}(111)$ substrate; (b) the interaction energy between graphene and the $\operatorname{Ir}(111)$ substrate, as well as the strain energy in graphene; (c) the charge transfer distribution in the (001) plane of the graphene/ Ir(111) moiré superstructure; and (d) the $\mathrm{C}-\mathrm{C}$ bond length distributions.
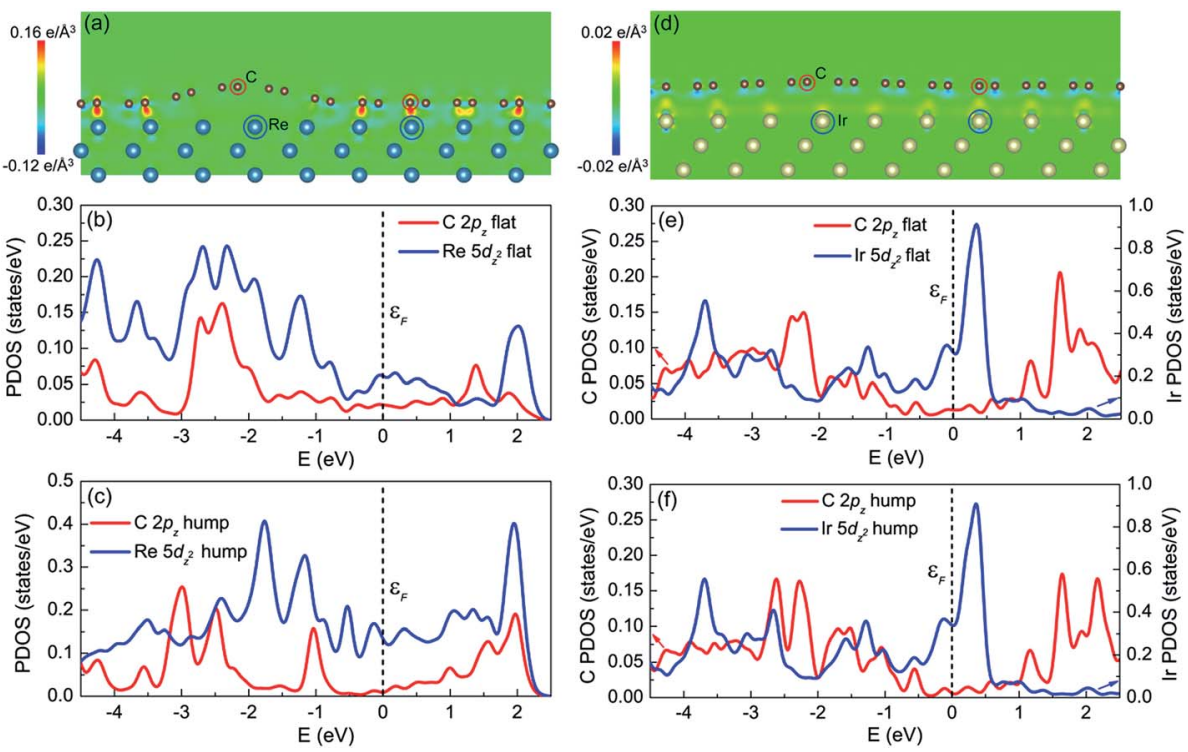

Fig. 5 (a) The interfacial charge-transfer distribution between graphene and Re(0001) substrate in the $10 \times 9$ model. (b) and (c) are the partial density of state (PDOS) for the labeled $\mathrm{C}$ atoms ( $2 \mathrm{p}_{z}$ orbital) and Re atoms $\left(5 \mathrm{~d}_{z^{2}}\right.$ orbital) in (a). (d) The interfacial charge-transfer distribution between graphene and the $\operatorname{Ir}(111)$ substrate. (e) and ( $\mathrm{f})$ are the partial density of states for the labeled $C$ atoms ( $2 \mathrm{p}_{z}$ orbital) and Ir atoms $\left(5 \mathrm{~d}_{z^{2}}\right.$ orbital) in (d).

\section{Conclusions}

In summary, we performed DFT calculations to study the graphene/Re(0001) and graphene/Ir(111) moiré superstructures. By comparing the interaction energy between graphene and the $\operatorname{Re}(111)$ substrate, as well as the strain energy of graphene in the two experimentally reported graphene/Re(0001) moire superstructures, we found that they are deeply influenced by the covalent interfacial interactions. Compared to the straindriven weak van der Waals interacting graphene/Ir(111) moiré superstructure, we found that interfacial interactions dominate the moire superstructure of graphene on the covalently interacting $\operatorname{Re}(0001)$ substrate. The strong interfacial interaction causes the deviation of $\mathrm{C}-\mathrm{C}$ bond lengths from those of ideal graphene, but with significant gain in the interfacial interaction energy. The strong covalent interaction between graphene and the $\operatorname{Re}(0001)$ substrate is closely related to the hybridization between the $\mathrm{C} 2 \mathrm{p}_{z}$ orbital and $\mathrm{Re} 5 \mathrm{~d}_{z^{2}}$ orbital. This study, therefore, provides a deeper understanding of the moire superstructures between graphene and the transition-metal substrates. 


\section{Acknowledgements}

The authors would like to acknowledge the support of the National Natural Science Foundation of China (Grant No. 51422504, 51527901, 51375010, 51501012). Simulations were carried out using the "Explorer 100" cluster system of the Tsinghua National Laboratory for Information Science and Technology.

\section{References}

1 W. Yang, G. Chen, Z. Shi, C. C. Liu, L. Zhang, G. Xie, M. Cheng, D. Wang, R. Yang, D. Shi, K. Watanabe, T. Taniguchi, Y. Yao, Y. Zhang and G. Zhang, Nat. Mater., 2013, 12, 792-797.

2 R. Balog, B. Jørgensen, L. Nilsson, M. Andersen, E. Rienks, M. Bianchi, M. Fanetti, E. Lægsgaard, A. Baraldi, S. Lizzit, Z. Sljivancanin, F. Besenbacher, B. Hammer, T. G. Pedersen, P. Hofmann and L. Hornekær, Nat. Mater., 2010, 9, 315-319.

3 C. R. Woods, L. Britnell, A. Eckmann, R. S. Ma, J. C. Lu, H. M. Guo, X. Lin, G. L. Yu, Y. Cao, R. V. Gorbachev, A. V. Kretinin, J. Park, L. A. Ponomarenko, M. I. Katsnelson, Yu. N. Gornostyrev, K. Watanabe, T. Taniguchi, C. Casiraghi, H.-J. Gao, A. K. Geim and K. S. Novoselov, Nat. Phys., 2014, 10, 451-456.

4 W. Ouyang, M. Ma, Q. Zheng and M. Urbakh, Nano Lett., 2016, 16(3), 1878-1883.

5 D. Deng, K. S. Novoselov, Q. Fu, N. Zheng, Z. Tian and X. Bao, Nat. Nanotechnol., 2016, 11, 218-230.

6 H. Tetlow, J. Posthuma de Boer, I. J. Ford, D. D. Vvedensky, J. Coraux and L. Kantorovich, Phys. Rep., 2014, 542, 195-295.

7 L. Meng, R. Wu, L. Zhang, L. Li, S. Du, Y. Wang and H.-J. Gao, J. Phys.: Condens. Matter, 2012, 24, 314214.

8 A. T. N'Diaye, S. Bleikamp, P. J. Feibelman and T. Michely, Phys. Rev. Lett., 2006, 97, 215501.

9 M. Gao, Y. Pan, L. Huang, H. Hu, L. Z. Zhang, H. M. Guo, S. X. Du and H.-J. Gao, Appl. Phys. Lett., 2011, 98, 033101.

10 P. Sutter, J. T. Sadowski and E. Sutter, Phys. Rev. B: Condens. Matter Mater. Phys., 2009, 80, 245411.

11 S. Nie, N. C. Bartelt, J. M. Wofford, O. D. Dubon, K. F. McCarty and K. Thürmer, Phys. Rev. B: Condens. Matter Mater. Phys., 2012, 85, 205406.
12 Y. Pan, H. Zhang, D. Shi, J. Sun, S. Du, F. Liu and H. Gao, Adv. Mater., 2009, 21, 2777-2780.

13 P. W. Sutter, J. I. Flege and E. A. Sutter, Nat. Mater., 2008, 7, 406-411.

14 L. Gao, Y. Liu, T. Ma, R. Shi, Y. Hu and J. Luo, Appl. Phys. Lett., 2016, 108, 261601.

15 D. Jiang, M. H. Du and S. Dai, J. Chem. Phys., 2009, 130, 074705.

16 B. Wang, M. L. Bocquet, S. Marchini, S. Günther and J. Wintterlin, Phys. Chem. Chem. Phys., 2008, 10, 3530-3534.

17 C. Tonnoir, A. Kimouche, J. Coraux, L. Magaud, B. Delsol, B. Gilles and C. Chapelier, Phys. Rev. Lett., 2013, 111, 246805.

18 E. Miniussi, M. Pozzo, A. Baraldi, E. Vesselli, R. R. Zhan, G. Comelli, T. O. Menteș, M. A. Niño, A. Locatelli, S. Lizzit and D. Alfè, Phys. Rev. Lett., 2011, 106, 216101.

19 P. Merino, M. Švec, A. L. Pinardi, G. Otero and J. A. MartínGago, ACS Nano, 2011, 5(7), 5627-5634.

20 G. Kresse and J. Furthmüller, Phys. Rev. B: Condens. Matter Mater. Phys., 1996, 54, 11169.

21 J. Klimes, D. R. Bowler and A. Michelides, J. Phys.: Condens. Matter, 2010, 22, 022201.

22 J. Klimes, D. R. Bowler and A. Michelides, Phys. Rev. B: Condens. Matter Mater. Phys., 2011, 83, 195131.

23 T. Björkman, J. Chem. Phys., 2014, 141, 074708.

24 M. Vanin, J. J. Mortensen, A. K. Kelkkanen, J. M. GarciaLastra, K. S. Thygesen and K. W. Jacobsen, Phys. Rev. B: Condens. Matter Mater. Phys., 2010, 81, 081408(R).

25 P. A. Khomyakov, G. Giovannetti, P. C. Rusu, G. Brocks, J. van den Brink and P. J. Kelly, Phys. Rev. B: Condens. Matter Mater. Phys., 2009, 79, 195425.

26 L. Z. Zhang, S. X. Du, J. T. Sun, L. Huang, L. Meng, W. Y. Xu, L. D. Pan, Y. Pan, Y. L. Wang, W. A. Hofer and H.-J. Gao, Adv. Mater. Interfaces, 2014, 1, 1300104.

27 A. L. Hsu, R. J. Koch, M. T. Ong, W. Fang, M. Hofmann, K. K. Kim, T. Seyller, M. S. Dresselhaus, E. J. Reed, J. Kong and T. Palacios, ACS Nano, 2014, 8, 7704-7713.

28 A. Dahal and M. Batzill, Nanoscale, 2014, 6, 2548-2562.

29 J. I. Martínez, P. Merino, A. L. Pinardi, O.-I. Gonzalo, M. F. López, J. Méndez and J. A. Martín-Gago, Sci. Rep., 2016, 6, 20354. 